\title{
Hepatic Cyst Infection Following Aspiration Sclerotherapy: a Case Series
}

\author{
Titus F.M. Wijnands ${ }^{\star}$, Marten A. Lantinga ${ }^{\star}$, Joost P.H. Drenth
}

Department of

Gastroenterology and

Hepatology, Radboud

University Medical Center,

Nijmegen,

The Netherlands

\author{
Address for correspondence: \\ Joost PH Drenth, M.D. Ph.D. \\ Professor of Gastroenterology \\ and Hepatology \\ Radboud University Medical \\ Center \\ P.O. Box 9101 \\ 6500 HB Nijmegen, \\ The Netherlands \\ joostphdrenth@cs.com
}

Received: 31.10 .2014 Accepted: 21.11.2014

\begin{abstract}
Mass-related symptoms caused by hepatic cysts are effectively treated by aspiration sclerotherapy (AS). This minimal-invasive intervention is regarded as a safe procedure. Hence, occurrence of complications is low. Transient fever is commonly reported as a side effect. However, documentation on a post-procedural hepatic cyst infection as a complication of AS is limited. We present five cases in which a tentative diagnosis of postprocedural hepatic cyst infection was made. Patients typically presented with abdominal pain and fever, had to be admitted to our hospital, and were treated with long term antibiotics. Ultimately, the cyst infection successfully resolved with ciprofloxacin in all cases.
\end{abstract}

Key words: aspiration sclerotherapy - post-procedural hepatic cyst infection - complication.

\section{INTRODUCTION}

Hepatic cysts are benign, fluid-filled cavities located in the liver that are estimated to occur in $2.5-11.3 \%$ of the general population $[1,2]$. Over lifetime, cysts tend to increase in volume, leading to mass-related symptoms requiring treatment [3]. Aspiration sclerotherapy (AS) is a minimal-invasive treatment option that curtails hepatic cyst volume and thereby alleviates mass-related symptoms [4]. Aspiration sclerotherapy is a procedure that comprises ultrasonography (US) guided percutaneous drainage of the hepatic cyst with subsequent instillation of a sclerosing agent. Sclerotherapy induces lysis of secreting cholangiocytes that cover the inner cyst wall [5]. The literature describes the use of many different sclerosing agents but ethanol is most commonly applied as it is effective, safe, inexpensive, and widely available [6-8]. The most common side effect of AS is pain during or after the procedure [3, 9, 10]. Other systemic complications include transient fever, ethanol intoxication, and pleural effusion $[8,11,12]$. Local complications resulting from AS, such as cyst infections, have only incidentally been documented. Spontaneous hepatic cyst infection is a severe complication that frequently necessitates invasive treatment and may even lead to sepsis or death $[13,14]$.

Over the past ten years we have performed over 250 AS procedures and encountered a number of hepatic cyst infections. This paper highlights five cases of post-procedural hepatic cyst infection to better understand the natural history of this complication, in order to provide therapeutical guidance.

\section{CASE 1}

A 59-year-old female with a history of polycystic liver disease presented with increasing abdominal pain. Ultrasonography showed multiple large hepatic cysts and AS was suggested in order to reduce mass-related symptoms. Without the administration of antibiotic prophylaxis, two large hepatic cysts were drained ( 5 Fr pigtail catheter). The first cyst located posteriorly in the right hepatic lobe contained $800 \mathrm{ml}$ of serous fluid, the second cyst located anteriorly in the left lobe contained $1500 \mathrm{ml}$ of brown, turbid fluid. One month following the procedure, the patient was seen in the emergency room with complaints of right-sided flank pain, fever and chills. Laboratory analysis showed an increased serum C-reactive protein (CRP) of $100 \mathrm{mg} / \mathrm{L}$ (Fig. 1). Ultrasonography revealed debris in the anterior hepatic cyst. Under the suspicion of a hepatic cyst infection, clindamycin (300 mg three times daily) 


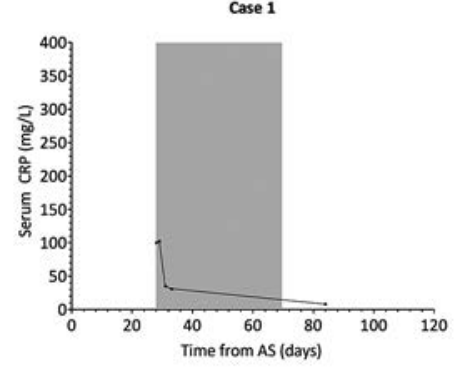

Case 3

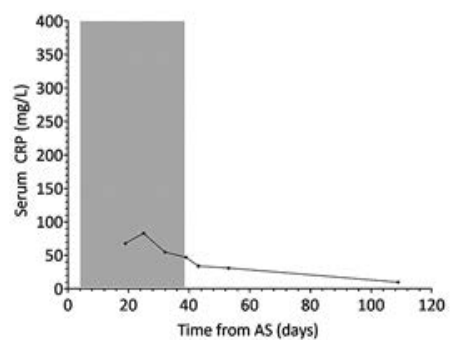

Case 2

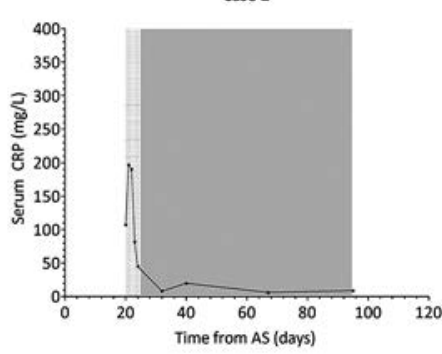

Case 4

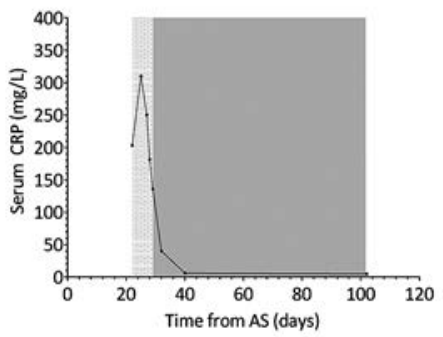

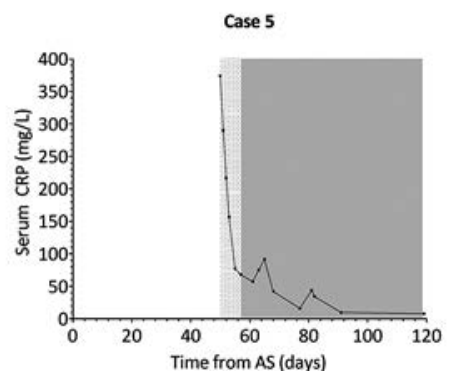

Fig. 1. Serum C-reactive protein (CRP) in response to antibiotic treatment (Cases 1-5). Filled areas correspond with intravenous (dotted) or oral (solid) antibiotic therapy.

and ciprofloxacin (500 mg twice daily) oral antibiotic therapy was initiated (Table I). During the next days, symptoms resolved and the patient was discharged. Clindamycin and ciprofloxacin were continued for a total of six weeks. Two weeks after the stopping of antibiotics, the serum CRP had returned to normal $(8 \mathrm{mg} / \mathrm{L})$.

\section{CASE 2}

A 54-year-old woman with a history of multiple hepatic cysts was referred to our outpatient clinic with complaints of upper abdominal pain. The largest hepatic cyst $(7 \mathrm{~cm})$ was located cranially in the right hepatic lobe. Because of continuous pain, AS was performed. During the procedure the catheter dislocated after aspiration of $200 \mathrm{ml}$ of clear cyst fluid. The intervention was discontinued prior to administration of ethanol. Initially, the patient experienced a relief of pain. However, three weeks later, upper abdominal pain and fever $\left(38.8^{\circ} \mathrm{C}\right)$ ensued. Laboratory analysis revealed increased serum CRP (107 mg/L) and white blood cell count $\left(13.2 \times 10^{9} / \mathrm{L}\right)$. Ultrasonography showed recurrence of the treated cyst with internal debris. The patient was admitted and treated with intravenous amoxicillin/clavulanic acid antibiotic therapy. After four days, antibiotic therapy was switched to oral moxifloxacin for six weeks (400 mg daily). This was later switched to ciprofloxacin (500 mg twice daily) for four additional weeks which led to a resolution of symptoms and normalization of serum CRP.

Table I. Clinical presentation and treatment of post-procedural hepatic cyst infection cases

\begin{tabular}{lcccc}
\hline Case & Age/sex & $\begin{array}{c}\text { Time between AS } \\
\text { and symptoms }\end{array}$ & Symptoms & Treatment \\
\hline 1 & $59 / \mathrm{F}$ & 4 weeks & Pain, fever and chills & Oral antibiotics \\
2 & $54 / \mathrm{F}$ & 3 weeks & Pain and fever & Intravenous and oral antibiotics \\
3 & $55 / \mathrm{F}$ & 3 days & Fever and chills & Oral antibiotics \\
4 & $57 / \mathrm{F}$ & 3 weeks & Pain and fever & $\begin{array}{c}\text { Intravenous and oral antibiotics } \\
\text { Cyst drainage }+ \text { intravenous and } \\
\text { oral antibiotics }\end{array}$ \\
\hline
\end{tabular}

AS, aspiration sclerotherapy; F, female 


\section{CASE 3}

A 55-year-old Caucasian female with a solitary hepatic cyst complained of increased abdominal pain and early satiety. A hydatid cyst was excluded during the initial workup. Ultrasonography showed a solitary hepatic cyst in the left hepatic lobe with a diameter of $18 \mathrm{~cm}$. Aspiration sclerotherapy was performed and $2500 \mathrm{ml}$ of clear fluid were aspirated. After the procedure, the patient became febrile $\left(38^{\circ} \mathrm{C}\right)$ without pain or other complaints. The next day, body temperature had normalized and the patient was discharged. Three days later, the patient presented with fever $\left(38.5^{\circ} \mathrm{C}\right)$ and chills. Ciprofloxacin (500 mg twice daily) for 14 days was started under the suspicion of a cyst infection. Due to a persisting elevated serum CRP (68 mg/L), ciprofloxacin was continued for another 30 days. Although serum CRP remained elevated (47 mg/L), ciprofloxacin was ultimately stopped. During an outpatient clinic visit two months later, the patients' symptoms had resolved and serum CRP had decreased to $10 \mathrm{mg} / \mathrm{L}$.

\section{CASE 4}

A 57-year-old woman with polycystic liver disease was seen in our outpatient clinic with continuous right flank pain since several months. Previous AS treatment of three hepatic cysts had led to a successful reduction of cyst volume and complaints. Recent computed tomography showed a dominant hepatic cyst of $9 \mathrm{~cm}$ seated cranially in the right hepatic lobe. The patient was therefore again referred for AS. Drainage yielded hemorrhagic cyst fluid and as active bleeding could not be ruled out, sclerosing with ethanol was not performed. Three weeks later, the patient presented with upper abdominal pain and fever $\left(39.9^{\circ} \mathrm{C}\right)$. Laboratory analysis showed an elevated serum CRP of $203 \mathrm{mg} / \mathrm{L}$. Blood cultures returned negative. ${ }^{18} \mathrm{~F}-\mathrm{FDG}$ PET/CT revealed increased metabolic activity at the aspirated hepatic cyst, suggesting cyst infection (Fig. 2). We initiated ceftriaxone and as the patient rapidly improved we switched to oral ciprofloxacin. After 12 weeks of treatment, the patient had completely recovered with normalized CRP $(5 \mathrm{mg} / \mathrm{L})$

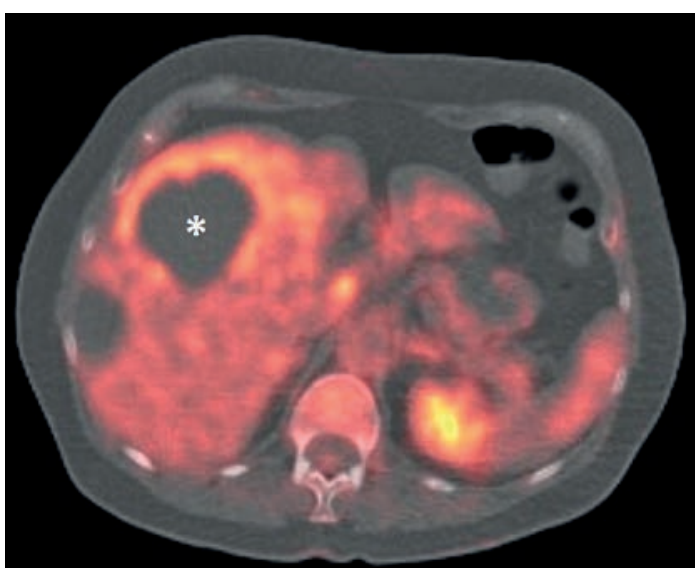

Fig. 2. ${ }^{18} \mathrm{~F}-\mathrm{FDG}$ PET/CT shows increased metabolic activity surrounding the previously aspirated hepatic cyst located in the right hepatic lobe (asterisk).

\section{CASE 5}

A 69-year-old female was referred to our clinic because a cardiological work-up for chest pain had led to the discovery of multiple hepatic cysts. Ultrasonography revealed a large cyst $(21 \mathrm{~cm})$ located in the right hepatic lobe. During AS, 3000 $\mathrm{ml}$ of clear, yellow fluid was drained. Seven weeks later, the patient complained of diarrhea ( $>10$ times daily), nausea, and vomiting ( $>5$ times daily). Physical examination revealed right and left upper quadrant abdominal tenderness and a subfebrile body temperature $\left(38.1^{\circ} \mathrm{C}\right)$. Laboratory investigations showed a highly increased serum CRP of $374 \mathrm{mg} / \mathrm{L}$. To support the diagnosis of a cyst infection in this patient, the recently aspirated cyst was punctured with aspiration of pus. A pigtail drain (10.5 Fr) was inserted to allow complete drainage of the cyst during the following days (Fig. 3). Whereas blood cultures remained negative, cyst aspirate culture grew, Klebsiella pneumoniae confirming cyst infection diagnosis. Intravenous ceftriaxone/metronidazole was started. During the next week, serum CRP gradually decreased to $68 \mathrm{ml} / \mathrm{L}$. The drain was removed and antibiotic therapy was switched to oral treatment with ciprofloxacin. After eight weeks of treatment symptoms resolved and serum CRP normalized.

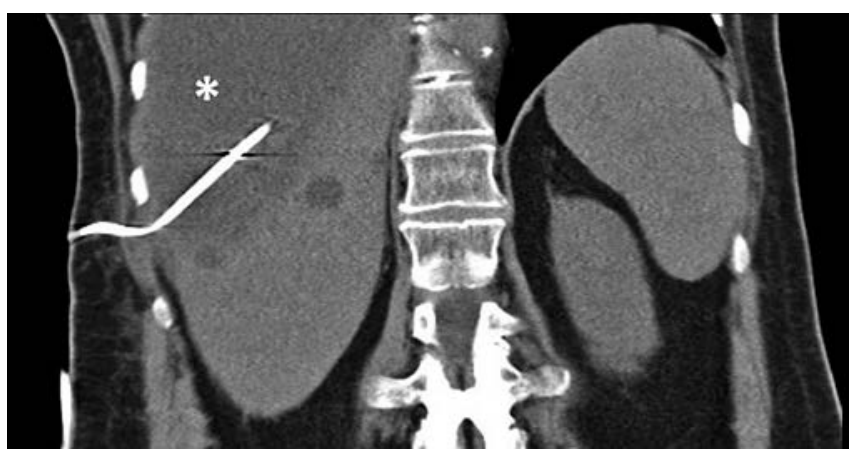

Fig. 3. Computed tomography demonstrating a large hepatic cyst in the right hepatic lobe (asterisk) with a pigtail drain inserted.

\section{DISCUSSION}

We describe the spectrum of inflammatory complications of AS that led to the tentative diagnosis of post-procedural hepatic cyst infection. All patients presented with acute onset abdominal pain and fever three days to seven weeks following AS. All patients required hospitalization, received prolonged antibiotic therapy (4-10 weeks), and recovered without any sequela.

An extensive literature search identified two cases of postprocedural hepatic cyst infection $[12,15]$. These case reports lack diagnostic findings that support hepatic cyst infection diagnosis, such as raised inflammatory markers, positive imaging or microbiological findings. Alternatively, the rise in body temperature directly following AS could be the result of an inflammatory response of the cystic wall, rather than a sign of post-procedural infection [16]. Indeed, transient fever is a commonly reported side effect $[10,17]$. However, both cases required intravenous antibiotic treatment $[12,15]$ suggesting a diagnosis of post-procedural hepatic cyst infection. 
The proposed gold standard in diagnosing a cyst infection is the presence of a cyst aspirate containing neutrophils and pathogens [13]. In absence of a cyst aspirate, a mix of clinical and biochemical findings are used to establish a probable cyst infection diagnosis [13]. In hindsight, four out of our five patients were labeled with the diagnosis of probable hepatic cyst infection. Treatment of cyst infection is empiric and primarily consists of long term antibiotic treatment [18]. In cases of failure, invasive strategies such as prolonged cyst drainage or even partial hepatectomy have been described as a management option [18]. In our experience, prolonged treatment with oral ciprofloxacin successfully resolved the episode.

To minimize the risk of infection, AS is performed in an interventional suite to provide a sterile environment. One hour prior to AS we administer cefazolin (intravenous, $1000 \mathrm{mg}$ ). Dutch guidelines recommend cefazolin, a first-generation cephalosporin, for perioperative prophylaxis [19]. Cefazolin is selectively active against micro-organisms most frequently isolated from surgical site infections and has a favourable plasma-half-life which allows single-dosing. Cefazolin is not used as a therapeutic agent [19]. Nonetheless, in our series a post-procedural hepatic cyst infection developed despite antibiotic prophylaxis. This could be explained by an inadequate bactericidal intracystic concentration, as it is unknown if cefazolin is able to penetrate into the cyst. Future studies investigating the penetration capacity of cefazolin into hepatic cysts would be valuable. Alternatively, the antimicrobial spectrum of first-generation cephalosporins might not cover the micro-organisms that caused the infection.

\section{CONCLUSION}

We presented five cases with inflammatory complications following AS, in which a post-procedural hepatic cyst infection was suspected. Antibiotic prophylaxis with cefazolin is unsuccessful in preventing cyst infection. In cases where a postprocedural hepatic cyst infection is suspected, we recommend pragmatic treatment with ciprofloxacin.

Consent: Written informed consent was obtained from all patients for publication of these cases and any accompanying images.

Conflicts of interest: The authors declare that they have no competing interests, support or funding.

Authors' contributions: T.W. and M.L. identified the cases, performed the literature search, and drafted the manuscript. J.D. participated in the design of the study, study coordination, and critically revised the manuscript. All authors read and approved the final manuscript.

\section{REFERENCES}

1. Gaines PA, Sampson MA. The prevalence and characterization of simple hepatic cysts by ultrasound examination. Br J Radiol 1989;62:335-337.
2. Larssen TB, Rorvik J, Hoff SR, Horn A, Rosendahl K. The occurrence of asymptomatic and symptomatic simple hepatic cysts. A prospective, hospital-based study. Clin Radiol 2005;60:1026-1029.

3. Drenth JP, Chrispijn M, Nagorney DM, Kamath PS, Torres VE. Medical and surgical treatment options for polycystic liver disease. Hepatology 2010;52:2223-2230.

4. Gevers TJ, Drenth JP. Diagnosis and management of polycystic liver disease. Nat Rev Gastroenterol Hepatol 2013;10:101-108.

5. Bean WJ, Rodan BA. Hepatic cysts: treatment with alcohol. AJR Am J Roentgenol 1985;144:237-241.

6. Benzimra J, Ronot M, Fuks D, et al. Hepatic cysts treated with percutaneous ethanol sclerotherapy: time to extend the indications to haemorrhagic cysts and polycystic liver disease. Eur Radiol 2014;24:1030-1038.

7. Montorsi M, Torzilli G, Fumagalli U, et al. Percutaneous alcohol sclerotherapy of simple hepatic cysts. Results from a multicentre survey in Italy. HPB Surg 1994;8:89-94.

8. Gelczer RK, Charboneau JW, Hussain S, Brown DL. Complications of percutaneous ethanol ablation. J Ultrasound Med 1998;17:531-533.

9. van Keimpema L, de Koning DB, Strijk SP, Drenth JP. Aspirationsclerotherapy results in effective control of liver volume in patients with liver cysts. Dig Dis Sci 2008;53:2251-2257.

10. Kairaluoma MI, Leinonen A, Stahlberg M, Paivansalo M, Kiviniemi $\mathrm{H}$, Siniluoto T. Percutaneous aspiration and alcohol sclerotherapy for symptomatic hepatic cysts. An alternative to surgical intervention. Ann Surg 1989;210:208-215.

11. Yamada N, Shinzawa H, Ukai K, et al. Treatment of symptomatic hepatic cysts by percutaneous instillation of minocycline hydrochloride. Dig Dis Sci 1994;39:2503-2509.

12. vanSonnenberg E, Wroblicka JT, D‘Agostino HB, et al. Symptomatic hepatic cysts: percutaneous drainage and sclerosis. Radiology 1994;190:387-392.

13. Lantinga MA, Drenth JP, Gevers TJ. Diagnostic criteria in renal and hepatic cyst infection. Nephrol Dial Transplant 2014 Jun 20.

14. Bleeker-Rovers CP, de Sevaux RGL, van Hamersvelt HW, Corstens FH, Oyen WJ. Diagnosis of renal and hepatic cyst infections by 18-F-fluorodeoxyglucose positron emission tomography in autosomal dominant polycystic kidney disease. Am J Kidney Dis 2003;41:E18-E21.

15. Erdogan D, van Delden OM, Rauws EA, et al. Results of percutaneous sclerotherapy and surgical treatment in patients with symptomatic simple liver cysts and polycystic liver disease. World J Gastroenterol 2007;13:3095-3100.

16. Larssen TB, Rorvik J, Horn A, et al. Biochemical and cytologic analysis of cystic contents in benign non-parasitic symptomatic hepatic cysts before and after ethanol sclerotherapy. Acta Radiol 2004;45:504-509.

17. Okano A, Hajiro K, Takakuwa H, Nishio A. Alcohol sclerotherapy of hepatic cysts: its effect in relation to ethanol concentration. Hepatol Res 2000;17:179-184.

18. Sallee M, Rafat C, Zahar JR, et al. Cyst infections in patients with autosomal dominant polycystic kidney disease. Clin J Am Soc Nephrol 2009;4:1183-1189.

19. van Kasteren ME, Gyssens IC, Kullberg BJ, Bruining HA, Stobberingh EE, Goris RJ. Optimizing antibiotics policy in the Netherlands. V. SWAB guidelines for perioperative antibiotic prophylaxis. Foundation Antibiotics Policy Team. Ned Tijdschr Geneeskd 2000;144:2049-2055. 